\title{
Influência do pilates na flexibilidade, sintomatologia dolorosa e na capacidade funcional em idosas
}

\author{
Pilates influence on flexibility, painful symptoms and functional capacity in older women \\ La influencia de pilates en la flexibilidad, los síntomas dolorosos y la capacidad funcional \\ en mujeres mayores
}

Felipe Santiago Oliveira Alves ${ }^{2}$, Jean Claude Lafetá ${ }^{1,2 *}$, Geraldo Magela Durães ${ }^{1,2}$, Maria de Fátima de Matos Maia ${ }^{1,2}$.

\section{RESUMO}

Objetivo: Verificar a influência do Pilates na flexibilidade, na sintomatologia dolorosa e na capacidade funcional de idosas. Métodos: Trata-se de uma pesquisa descritiva, com corte transversal e quantitativa. Participaram da amostra 90 idosas, com idade entre 65 a 75 anos, subdivididas aleatoriamente nos grupos G1 (Não praticantes de Exercicío Físico-EF), G2 (Pilates e outros EF) e G3 (Praticantes de outros EF), sendo submetidas ao Inventário de Dor de Back, análise da flexibilidade (Apley, sentar e alcançar-SA) e da capacidade funcional (levantar e caminhar-LC). $\mathrm{Na}$ análise dos dados recorreu-se à estatistica descritiva, correlação de Pearson, Anova Oneway e Kruskal-Wallis, com $p \leq 0,05$. Resultados: Evidenciou-se diferenças significativas na flexiblidade entre os grupos nos testes SA1 e SA2 $(p<0,001)$, com melhores resultados para o G2, embora não ocorrer no Apley direito $(p=0,391)$ e esquerdo $(p=0,076)$. A capacidade funcional apresentou-se com diferenças significativas entre grupos nos dois momentos $(p<0,001)$ investigados, da mesma forma que a sintomatologia dolorosa apresentada nos últimos três meses $(p=0,002)$, útimo mês e semana $(p<0,001)$. Conclusão: As idosas praticantes de exercício físico apresentaram melhores índices de flexibilidade, capacidade funcional e menores níveis de sintomatologia dolorosa do que as não praticantes, sendo que as participantes do Pilates demonstraram resultados mais satisfatórios.

Palavras-chave: Idosas, Método pilates, Flexibilidade, Funcionalidade, Percepção da dor.

\begin{abstract}
Objective: To verify the influence of Pilates on flexibility, painful symptoms and functional capacity of elderly women. Methods: This is a descriptive, cross-sectional and quantitative research. The sample consisted of 90 elderly women, aged 65 to 75 years, randomly subdivided into groups G1 (Non Physical Exercise-PE practitioners), G2 (Pilates and other PE) and G3 (Practitioners of other PE), undergoing the Back Pain Inventory. , analysis of flexibility (Apley, sitting and reaching-SA) and functional capacity (lifting and walking LC). In the data analysis we used the descriptive statistics, Pearson correlation, Anova Oneway and Kruskal Wallis, with $p<0.05$. Results: There were significant differences in flexibility between the groups in tests SA1 and SA2 $(p<0.001)$, with better results for $G 2$, although not in the right $(p=0.391)$ and left $(p=0.076)$ Apley. Functional capacity was significantly different between groups at the two time points $(p<0.001)$ investigated, as did the painful symptoms presented in the last three months $(p=0.002)$, last month and week $(p<0.001)$. Conclusion: The elderly women who practiced physical exercise had better flexibility, functional capacity and lower levels of pain symptoms than those who did not, and Pilates participants showed more satisfactory results.
\end{abstract}

Keywords: Elderly, Pilates method, Flexibility, Functionality, Pain perception.

1 Universidade Trás-dos-Montes e Alto Doro (UTAD). Vila Real - Portugal. *E-mail: jclafeta@yahoo.com.br

2 Universidade Estadual de Montes Claros (UNIMONTES). Montes Claros - MG.

SUBMETIDO EM: 5/2020

ACEITO EM: 6/2020

PUBLICADO EM: 9/2020

REAS/EJCH | Vol.12(11) | e2413 | DOI: https://doi.org/10.25248/reas.e2413.2020 Página 1 de 9 


\section{RESUMEN}

Objetivo: Verificar la influencia de Pilates sobre la flexibilidad, los síntomas dolorosos y la capacidad funcional de las mujeres mayores. Métodos: Esta es una investigación descriptiva, transversal y cuantitativa. La muestra consistió en 90 mujeres de edad avanzada, de 65 a 75 años, subdivididas aleatoriamente en grupos G1 (No practicantes de ejercicio fisico-PE), G2 (Pilates y otras PE) y G3 (practicantes de otras PE), sometidas al Inventario de Dolor de Espalda, el análisis de flexibilidad (Apley, sentado y alcanzando SA) y la capacidad funcional (levantar y caminar-LC). En el análisis de datos utilizamos la estadística descriptiva, la correlación de Pearson, Anova Oneway y Kruskal Wallis, con $p<0.05$. Resultados: Hubo diferencias significativas en la flexibilidad entre los grupos en las pruebas SA1 y SA2 $(p<0.001)$, con mejores resultados para G2, aunque no en la derecha $(p=0.391)$ e izquierda $(p=0.076)$ Apley. La capacidad funcional fue significativamente diferente entre los grupos en los dos puntos de tiempo $(p<0.001)$ investigados, al igual que los síntomas dolorosos presentados en los últimos tres meses $(p=0.002)$, el último mes y la semana $(p<0.001)$. Conclusión: Las mujeres de edad avanzada que practicaban ejercicio físico tenían una mejor flexibilidad, capacidad funcional y niveles más bajos de síntomas de dolor que aquellas que no lo hicieron, y los participantes de Pilates mostraron resultados más satisfactorios.

Palabras clave: Ancianos, Método pilates, Flexibilidad, Funcionalidad, Percepción del dolor.

\section{INTRODUÇÃO}

Várias mudanças têm ocorrido no Brasil relacionado ao perfil demográfico com a modificação da pirâmide etária. Antes considerado um país de jovens, hoje existe outra realidade, com um significativo acréscimo da sobrevida e a aumento da população idosa (WANDERLEY RL, et al., 2019). A terceira idade já ocupa um espaço significativo em nossa sociedade e continuará a aumentar nos próximos anos, ao ponto de brasileiros acima de 60 anos que em 2017 representava 13,8\% da população, em 2030 representará 18,6\% (VALDANHA NETO A, et al., 2017).

O desenvolvimento da capacidade funcional e aprimoramento da qualidade de vida são essenciais para se lidar com as atividades cotidianas dos idosos, visto que manter-se com a melhor aptidão física possível é um fator primordial para esse grupo etário. As atividades do dia-a-dia, tais como, ir às compras, levantar de uma cadeira, vestir, requerem um nível mínimo de força muscular, coordenação, flexibilidade e equilíbrio (VIEIRA SCAL, et al., 2015).

Com o aumento da expectativa de vida, as pessoas tendem a apresentar um índice maior de distúrbios álgicos e comorbidades, sendo muito frequentes as queixas dolorosas nos idosos, principalmente pelas dores crônicas, que contribuem negativamente na qualidade de vida e na redução da capacidade funcional (SANTOS FC, et al., 2012). Programas de saúde pública voltadas para as pessoas da terceira idade constituem um desafio, especialmente na qualificação de profissionais para uma equipe multidisciplinar, apoio de gestores, na sensibilização da população, com a elaboração de estratégias e a existência de políticas públicas especificas para esta população (FERREIRA OGI, et al., 2012).

Atualmente existem diversas modalidades de EF para o público idoso, como a hidroginástica, dança, pilates, natação, musculação, ioga, dentre outros. O Pilates vem ganhando cada vez mais espaço, por ser uma técnica eficaz na promoção da melhoria da capacidade funcional e atua com exercícios voltados para o equilíbrio estático. Por ser uma atividade física mais individualizada, vêm crescendo a sua procura visando a profilaxia de lesões para essa faixa etária (COSTA LMRD, et al., 2016).

Estudos descrevem que o Pilates promove diversos efeitos sobre a postura, flexibilidade, ganho de força, desenvolvimento da massa magra e do sistema cardiopulmonar (REIS LA, et al., 2011). No entanto, são limitadas no momento, as evidências que demonstrem a influência do Pilates na capacidade funcional dos idosos, principalmente relacionando a flexibilidade à sintomatologia dolorosa. Desde modo, esta investigação buscou averiguar a influência do Pilates na flexibilidade, sintomatologia dolorosa e na capacidade funcional em idosas. 


\section{MÉTODOS}

A pesquisa pode ser delineada como descritiva, transversal e com análise quantitativa dos dados. A população foi constituída por pessoas da terceira idade, do sexo feminino, residentes no município de Montes Claros - MG. A amostra foi composta por 90 idosas, com idade entre 65 a 75 anos, sendo selecionadas e subdivididas aleatoriamente em três grupos (G1 - não praticantes de EF regulares, G2 - praticantes de Pilates e outros EF e G3 - praticantes de outros EF).

Após a aprovação do Comitê de Ética e autorização das instituições que foram investigadas, todas as participantes foram submetidas ao preenchimento dos dados pessoais, anamnese (ficha de avaliação física) e ao questionário de distúrbios álgicos. Para averiguar a sintomatologia dolorosa foi aplicado o inventário de Dor de Becker Adaptado, que contém questões relacionadas à dor nos últimos três meses, no último mês e na última semana, à intensidade e ao local onde manifestava os distúrbios álgicos.

Para avaliar a flexibilidade da cadeia posterior foi utilizado o teste de sentar e alcançar (SA) os pés. $O$ avaliado sentava-se na borda da cadeira, uma das pernas ficava fletidas com os pés no chão, onde a outra era estendida ao máximo à frente do quadril, com o calcanhar no chão e pé fletido em aproximadamente $90^{\circ}$. Após os participantes terem praticado duas vezes com a perna selecionada, foram coletados em duas tentativas, registrando-se o escore em centímetros (MAZO GL, 2009; RICKLI RE e JONES CJ, 2008).

$\mathrm{Na}$ flexibilidade dos ombros, foi usado o teste de Apley (alcançar as costas), no qual a participante deveria realizar uma elevação do braço, flexionava o cotovelo, e tentava tocar a posição mais baixa possível, nas costas, com a palma da mão virada para o corpo. Ao mesmo tempo, realizava a extensão do outro braço para baixo, dobrava o cotovelo e, com a palma da mão para fora, aproximava ou tentava sobrepor os dedos da mão oposta. Para a mensuração, anotou a distância entre as mãos $(\mathrm{cm})$, atribuindo um sinal negativo ou positivo a esse valor (MAZO GL, 2009; RICKLI RE e JONES CJ, 2008).

Para análise da capacidade funcional das idosas foi utilizado o teste de levantar e caminhar (LC), que teve como objetivo avaliar a agilidade e o equilíbrio dinâmico. O avaliado começava em uma posição sentada na cadeira com uma postura ereta, com um pé levemente na frente do outro. Ao comando, levantava-se da cadeira, caminhava o mais rápido possível até o cone, contornava-o e voltava para a cadeira, sentando-se novamente. A pontuação foi registrada o tempo dos dois escores do teste para o décimo de segundo mais próximo (MAZO GL, 2009; RICKLI RE e JONES CJ, 2008).

Os dados foram analisados no SPSS (versão 20.0), sendo realizada a estatística descritiva, com média, desvio padrão, percentagens e frequências. Para normalidade da amostra recorreu-se ao teste Shapiro Wilk, já nas variáveis independentes aos testes Anova One Way, Kruskal-Wallis e Pós-hoc de Scheffe. Na análise da associação entre flexibilidade, capacidade funcional e algia utilizou-se da correlação de Pearson, com nível de significância de $5 \%$.

Esta investigação foi pautada na Resolução № 466, de 12 e Dezembro de 2012 do Conselho Nacional de Saúde (CNE), sendo submetida e apreciada pelo Comitê de Ética em Pesquisa da Universidade Estadual de Montes Claros - UNIMONTES, com aprovação através do Parecer № 3.331.510, autorizando assim, o envolvimento de seres humanos na realização da pesquisa.

\section{RESULTADOS}

Nesta investigação a amostra foi constitutida por 90 idosas, com média de idade de 69,3 $( \pm 3,24)$ anos, tendo o subgrupo de não participantes de $E F(G 1)$ média de $69,5( \pm 2,93)$ anos, o grupo de praticante de pilates e outros EF com média de $68,9( \pm 3,33)$ anos e os praticantes de outros EF com média de $69,6( \pm 3,38)$ anos.

Com relação a prática regular de EF ou modalidades esportivas, evidenciou-se que as idosas realizavam principalmente Pilates com 33,3\% ( $n=30)$, hidroginástica com 18,9\% ( $n=17)$, caminhada com 17,7\% $(n=16)$, musculação com 9,9\%(n=9) e condicionamento físico com $5,5 \%(n=5)$ da amostra. A maioria das participantes realizavam EF com frequencia semanal igual a 3,25 $( \pm 1,08)$ e duração de 60 minutos $(41,6 \%)$. No que se refere às patologias apresentadas, observou-se maior ocorrência da hipertensão arterial sistêmica 
(24,5\%), osteoartrose $(20,7 \%)$, hernia discal lombar $(13,2 \%)$, hernia cervical $(9,4 \%)$, lesão meniscal $(9,4 \%)$, sendo que grande parte $(41,1 \%, n=37)$ dos avaliados não relataram possuir nenhuma patologia.

A Tabela 1 demonstra a análise comparativa da flexibilidade entre os grupos investigados. Desta forma, verificou-se que não houve uma diferença significativa na flexiblidade da cintura escapular direita $(p=0,391)$ e esquerda $(p=0,076)$, embora os praticantes de pilates e de outros EF apresentaram uma melhor flexiblidade do que os não praticantes. Por outro lado, observou-se uma diferença muito significativa da flexibilidade da cadeia posterior entre os grupos demonstrado nos testes SA $1(p<0,001)$ e SA $2(p<0,001)$. No teste Post Hoc de Scheffe, observou-se uma diferença significativa dos grupos que realizavam EF em relação ao grupo que não realizava $(p<0,001)$. No entanto, embora o grupo do pilates apresentar melhores resultados, não ocorreu de forma expressiva em relação ao grupo de outros EF.

Tabela 1 - Análise do nível de flexibilidade entre os grupos investigados.

\begin{tabular}{|c|c|c|c|c|c|c|c|c|}
\hline Variável & $\mathrm{n}$ & $\begin{array}{c}\text { Não Pratica } \\
\text { EF }\end{array}$ & $\mathbf{n}$ & $\begin{array}{l}\text { Pilates e } \\
\text { outras EF }\end{array}$ & $\mathbf{n}$ & Pratica EF & $f$ & Sig \\
\hline & & Média/ DP & & Média/ DP & & Média/ DP & & \\
\hline Apley D & 30 & $-11,86 \pm 9,03$ & 30 & $6,16 \pm 12,56$ & 30 & $\begin{array}{c}-6,23 \pm \\
11,10\end{array}$ & 2,655 & 0,391 \\
\hline Apley E & 30 & $-15,33 \pm 8,64$ & 30 & $-10,63 \pm 15,13$ & 30 & $\begin{array}{c}- \\
12,93 \pm 14 \\
82\end{array}$ & 0,96 & 0,076 \\
\hline $\begin{array}{c}\text { Sentar e } \\
\text { Alcançar } 1\end{array}$ & 30 & $-8,40 \pm 7,31$ & 30 & $2,36 \pm 7,30$ & 30 & $\begin{array}{c}- \\
1,70 \pm 13,0 \\
1\end{array}$ & 9,633 & $\begin{array}{c}*<0,00 \\
1\end{array}$ \\
\hline $\begin{array}{c}\text { Sentar e } \\
\text { Alcançar } 2\end{array}$ & 30 & $-7,43 \pm 7,27$ & 30 & $3,30 \pm 7,62$ & 30 & $\begin{array}{c}0,43 \\
\pm 10,05\end{array}$ & 13,096 & $\begin{array}{c}{ }^{*}<0,00 \\
1\end{array}$ \\
\hline
\end{tabular}

Legenda: ${ }^{*} p \leq 0,05$. Fonte: Alves FSO, et al., 2019.

Na Tabela 2 fica evidenciado uma diferença significativa entre os grupos nos testes LC no primeiro momento $(p<0,001)$ e segundo momento $(p<0,001)$. Além disso, o Post Hoc revela que existe uma diferença significativa dos praticantes de EF comparando aos não praticantes, embora não apresentar diferença significativa entre os dois grupos que participavam de exercicios regulares.

Tabela 2 - Análise comparativa da capacidade funcional entre os grupos investigados.

\begin{tabular}{ccccccccc}
\hline Variável & $\mathbf{n}$ & Não Pratica EF & $\mathbf{n}$ & $\begin{array}{c}\text { Pilates e } \\
\text { outras EF }\end{array}$ & $\mathbf{n}$ & Pratica EF & $\mathbf{f}$ & Sig \\
\hline Média/ DP & & Média/ DP & & Média/ DP & & \\
\hline $\begin{array}{l}\text { Levantar e } \\
\text { caminhar 1 }\end{array}$ & 30 & $7,89 \pm 1,28$ & 30 & $5,61 \pm 1,47$ & 3 & $6,44 \pm 1,47$ & 19,926 & ${ }^{*}<0,00$ \\
$\begin{array}{l}\text { Levantar e } \\
\text { caminhar 2 }\end{array}$ & 30 & $7,46 \pm 1,18$ & 30 & $5,43 \pm 1,47$ & 3 & $6,47 \pm 1,53$ & 15,550 & ${ }^{*}<0,00$ \\
\end{tabular}

Legenda: ${ }^{*} p \leq 0,05$. Fonte: Alves FSO, et al., 2019.

Ao que se refere a ocorrência da sintomatologia dolorosa, as participantes do G1 relataram apresentar dor nos últimos três meses principalmente na parte anterior do corpo, no joelho direito (42\%) e joelho esquerdo (28\%), e na parte posterior corpórea, na região torocolombar $(75 \%)$ e no trapézio $(31,2 \%)$. As integrantes do 
G2 tambem relataram maior frenquência de dor nos ultimos tres meses na parte anterior, no joelho direito $(41,6)$ e esquerdo $(25 \%)$, e na parte posterior, na região toracolombar $(28,5 \%)$ e no ombro direito $(21,4 \%)$. Já as idosas do G3 relataram dor nos ultimos tres meses na parte anterior, na região do joelho esquerdo (16,6\%) e no quadril direito $(8,3 \%)$, e na parte posterior, na região toracolombar $(35,2 \%)$ e do trapezio $(17,6 \%)$.

Em relação aos segmentos mais afetados da sintomatologia dolorosa no último mês, as idosas do G1 relataram sentir dor na parte anterior, na clavicula $(18,1 \%)$ e no joelho direito $(9,9 \%)$, e na parte posterior, na região toracolombar $(68,7 \%)$ e trapezio bilateralmente $(31,2 \%)$. No G2, as integrantes relataram sentir dor no ultimo mês, na parte anterior, no joelho direito $(36,3 \%)$ e joelho esquerdo $(18,1 \%)$, e na parte posterior, na porção toracolombar (25\%) e ombro direito $(18,7 \%)$. Já as participantes do G3 relataram que sentiram mais dor na parte anterior, no joelho direito $(27,2 \%)$ e no punho direito $(9 \%)$, e na parte posterior, na porção toracolombar (43,7\%) e apenas torácica (12,5\%).

Referindo-se aos locais de dor na ultima semana, as integrantes do G1 relataram apresentar na parte anterior corpórea, na região clavicular $(20 \%)$ e joelho direito $(10 \%)$, e na parte posterior, na porção toracolombar $(75 \%)$ e trapézio $(31,2 \%)$ bilateralmente. As participantes do G2 relataram na parte anterior, no joelho direito $(30 \%)$ e esquerdo (30\%), e na parte posterior, no ombro direito $(18 \%)$ e toracolombar (12\%). No G3, relataram manifestações álgicas na parte anterior, no joelho direito $(20 \%)$ e punho direito $(10 \%)$, e na parte posterior, no segmento toracolombar $(25 \%)$ e trápezio $(25 \%)$ de forma bilateral.

Com relação à frequência da sintomatologia dolorosa, ficou evidenciado no G1, que nos últimos três meses, $50 \%$ dos participantes tiveram episódios de dor várias vezes ao dia, $20 \%$ mencionaram sentirem dor uma vez ao dia, sendo que no último mês, $46 \%$ aprensentaram dor várias vezes ao dia e $20 \%$ relataram dores uma vez ao dia. No G2, 36\% mencionaram sentirem dor várias vezes ao dia e $20 \%$ de forma esporádica nos últimos três meses, $16 \%$ apresentarm dor varias vezes ao dia e 30\% relaaram sentir dores de forma esporádica no ultimo mês. Já no G3, foi possível verificar que nos últimos três meses, $26,7 \%$ apresentaram dor várias vezes ao dia e $33,3 \%$ de forma esporádica, no último mês $20 \%$ apresentaram dor varias vezes ao dia e $33,3 \%$ relataram sentir dor de forma esporádica.

Na Tabela 3 evidenciou-se uma diferença muito significativa entre os grupos, tanto no índice álgico dos últimos três meses $(p=0,002)$, do último mês $(p<0,001)$ e semana $(p<0,001)$. Além disso,o Post Hoc expõe uma diferença significativa dos grupos G2 e G3 em relação ao G1 para a dor dos ultimos três meses $(p=0,022)$, no último mês $(p=0,001)$ e semana $(p<0,001)$, embora não apresentar diferença significativa entre o G2 e G3 da intensidade da dor dos ultimos três meses $(p=0,829)$, no último mês $(p=0,879)$ e semana $(p<0,599)$.

Tabela 3 - Análise comparativa do nível de intensidade álgica.

\begin{tabular}{|c|c|c|c|c|c|c|c|c|}
\hline Variável & $\mathbf{n}$ & $\begin{array}{c}\text { Não Pratica } \\
\text { EF }\end{array}$ & $\mathbf{n}$ & $\begin{array}{c}\text { Pilates e } \\
\text { outras } \\
\text { EF }\end{array}$ & $\mathbf{n}$ & $\begin{array}{l}\text { Pratica } \\
\text { EF }\end{array}$ & $f$ & Sig \\
\hline & & Média/ DP & & $\begin{array}{l}\text { Média/ } \\
\text { DP }\end{array}$ & & $\begin{array}{l}\text { Média/ } \\
\text { DP }\end{array}$ & & \\
\hline Intens. dor 3 meses & 30 & $5,96 \pm 3,07$ & 30 & $\begin{array}{c}3,66 \\
\pm 3,24\end{array}$ & 30 & $\begin{array}{c}3,16 \\
\pm 3,16\end{array}$ & 6,692 & ${ }^{*} 0,002$ \\
\hline Intens. dor do último mes & 30 & $6,00 \pm 2,70$ & 30 & $\begin{array}{r}3,20 \\
\pm 2,98\end{array}$ & 30 & $\begin{array}{r}2,83 \\
\pm 2,67\end{array}$ & 11,534 & ${ }^{*}<0,001$ \\
\hline $\begin{array}{l}\text { Intens. dor da última } \\
\text { semana }\end{array}$ & 30 & $5,83 \pm 2,75$ & 30 & $\begin{array}{r}1,66 \\
\pm 2,74\end{array}$ & 30 & $\begin{array}{c}2,40 \\
\pm 2,88\end{array}$ & 18,990 & ${ }^{*}<0,001$ \\
\hline
\end{tabular}

Legenda: ${ }^{*} p \leq 0,05$. Fonte: Alves FSO, et al., 2019.

Com a análise de correlação de Pearson, evidenciou-se uma associação entre a flexibilidade e a capacidade funcional e sintomatologia dolorosa das idosas, tendo em vista que foi observado uma fraca correlação negativa entre os niveis de flexibildade da cadeia posterior (momento 1) com a itensidade da dor 
averiguada nos ultimos 3 meses $(r=-0,227, p=0,032)$, no último mês ( $r=-0,252, p=0,016)$ e na última semana $(r=-0.263, p=0,01)$ e no teste de flexibilidade no momento dois, dos ultimos três meses $(r=-0,306, p=0,003)$, do último mês $(r=-0,332, p=0,001)$ e da ultima semana $(r=-0,318, p=0,003)$. Além disso, foi constatada uma fraca correlação negativa entre os niveis de flexibildade da cintura escapular (Apley do ombro D) com a intensidade da dor averiguada dos ultimos 3 meses $(r=-0,306, p=0,003)$, do último mês $(r=-0,345, p=0,001)$ e da última semana $(r=-0,341, p=0,001)$ e da flexibilidade do ombro $E$ com a itensidade da dor averiguada nos ultimos 3 meses $(r=-0,269, p=0,010)$, no último mês $(r=-0,261, p=0,013)$ e na última semana $(r=-0,275$, $p=0,009$ ). (Gráfico 1)

Gráfico 1 - A: Análise correlacional entre Flexibilidade - SA e Capacidade Funcional - LC. B: Flexibilidade SA e Algias.

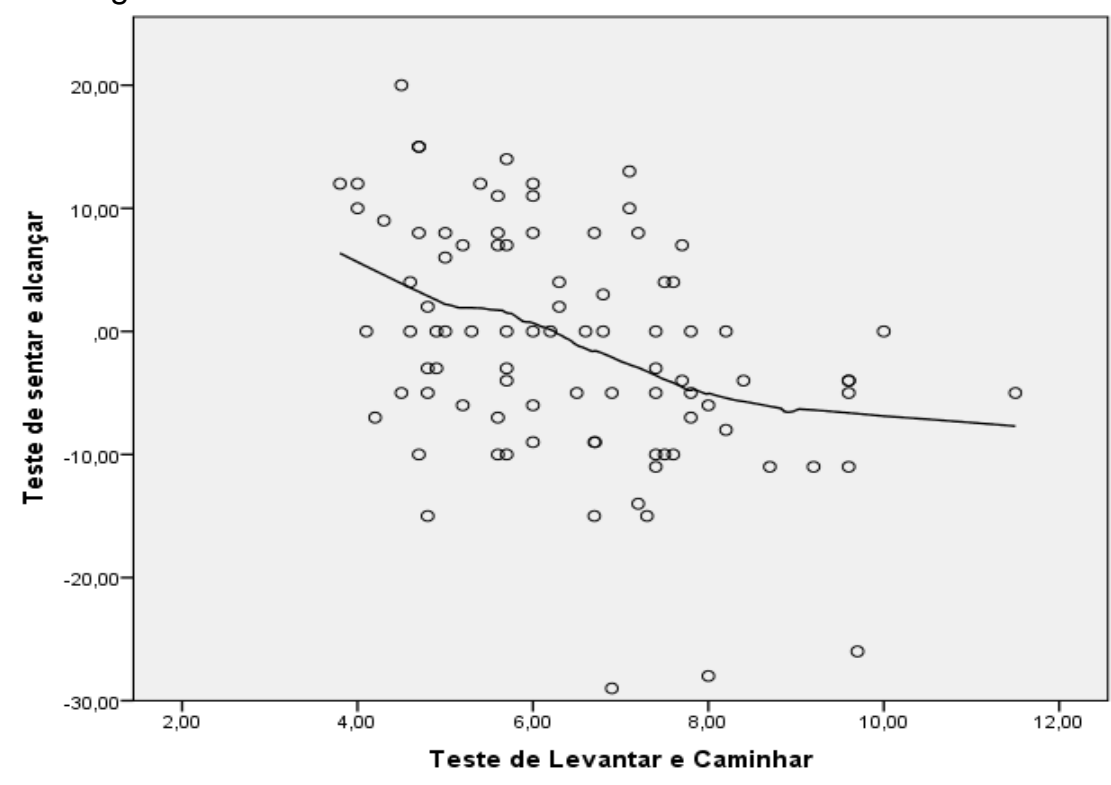

Gráfico A)

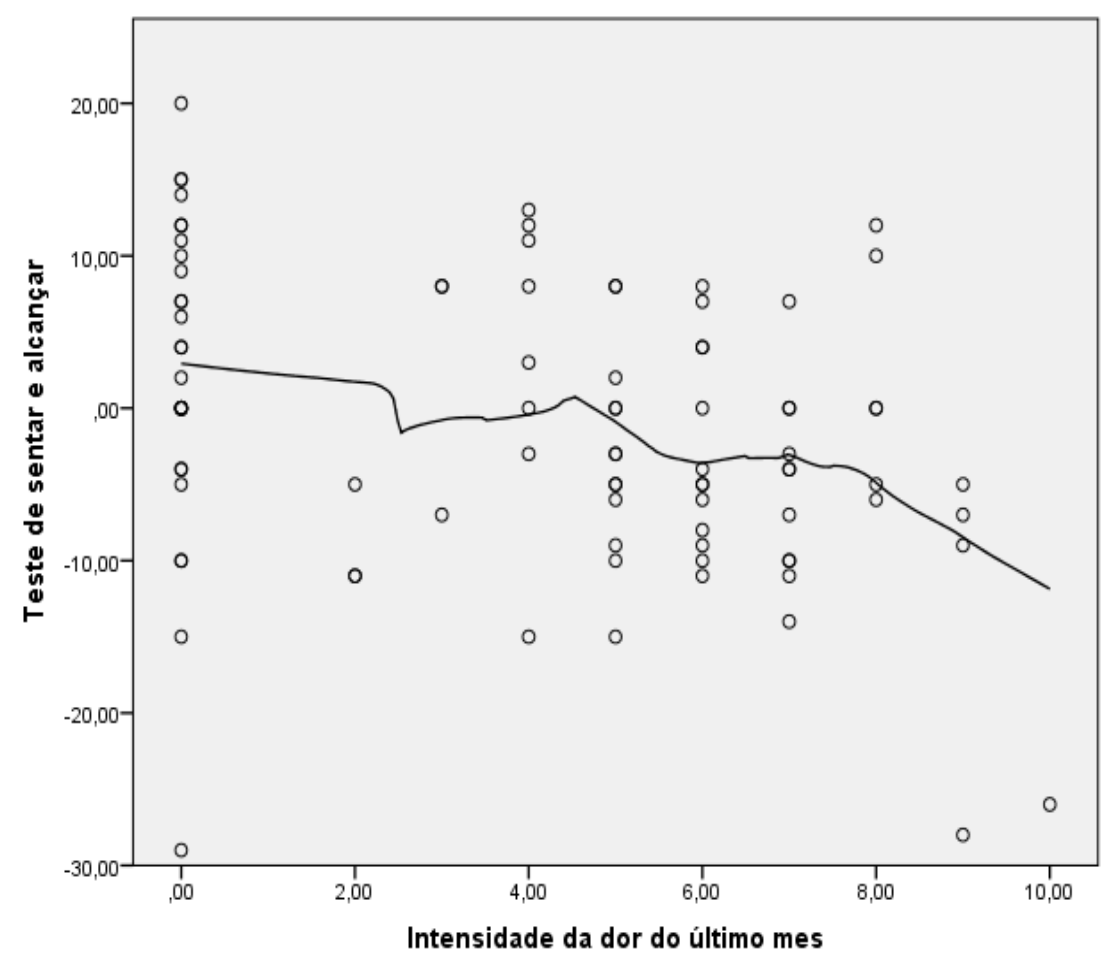

Gráfico B)

Fonte: Alves FSO, et al., 2019. 
Da mesma forma foi observado uma moderada correlação negativa da flexibilidade da cadeia posterior com a capacidade funcional das idosas, averiguado nos testes no momento um $(r=-0,396, p<0,001)$ e no momento dois $(r=-0,429, p<0,001)$. De forma identica, no segundo momento do teste de flexibilidade, contatou-se uma moderada correlação negativa entre a capacidade funcional examinada no primeiro momento $(r=-0,416, p<0,001)$ e segundo momento $(r=-0,423, p<0,001)$.

\section{DISCUSSÃO}

Os resultados coletados nesta pesquisa demonstram que as idosas apresentaram uma maior ocorrência de patologias cardiovasculares e osteomusculares como a hipertensão arterial sistêmica, osteoartrose, hernia discal e lesão meniscal. No entanto, grande parcela das entrevistadas não relataram apresentar doenças crônicas. Além disso, a maioria não fazem uso de farmacoterapia regularmente, enquanto as demais utilizam principalmente Losartana, Suplemento de Cálcio, Puratan e Paco.

Estes achados se assemelham à literatura, pois diversos estudos observaram nos idosos uma maior prevalência de condições patogênicas cardiovasculares e músculoesqueléticas (ÁVILA AF, et al., 2019). No entanto, para Ribeiro PCC et al. (2018), as patologias mais frequentes se diferenciam com relação ao sexo, ocorrendo principalmente nos homens as cardiopatias e câncer, e, nas mulheres, a artrite reumatóide, incontinência urinária, depressão e osteoporose. Contudo, a hipertensão é comumente apontada como a mais frequente.

No que se refere a flexibilidade, ficou evidenciado nesta investigação que idosas que participavam regularmente de EF (G2 e G3) apresentavam articulações da cadeia posterior bem mais flexíveis do que das não praticantes (G1), embora não ocorrer de forma significante na cintura escapular bilateral.

No entanto, as integrantes do G2 obtiveram melhores resultados do que G3, apesar de não ser tão expressivos. Portanto, participar regularmente de EF, principalmente associando diversas modalidades, tais como o Pilates, podem afetar positivamente na manutenção de uma flexibilidade adequada para a população da terceira idade.

Esses resultados corroboram com a literatura pertinente, pois Tozim BM et al. (2014), avaliaram os efeitos promovidos pelo Pilates sobre a flexibilidade, qualidade de vida e dor de idosas. Participaram desse estudo 31 idosas, subdivididas em um grupo Pilates $(n=14)$ e outro controle $(n=14)$, sendo a flexibilidade avaliada no pré e pós-teste (8 semanas) pelo teste de sentar e alcançar - SA e ângulo poplíteo. Com resultados semelhantes desse estudo, evidenciaram uma melhora significativa da flexibilidade no AS, ângulo poplíteo direito e esquerdo com a aplicação do método Pilates.

Em outro estudo Kneip $\mathrm{K}$ et al. (2018), averiguaram a influência do método Pilates sobre a flexibilidade dos membros inferiores de 15 idosos. Para tal, a flexibilidade foi mensurada com o banco de Wells antes e após o período de intervenção. Os resultados revelaram um aumento expressivo da flexibilidade de idosos sedentários, no qual os seus achados e metodologia para mensuração da flexibilidade foram parecidos como da presente investigação.

Irez GB (2014) realizou uma investigação para observar os benefícios de um programa de Pilates solo para componentes motores e prevenção de quedas em idosos. Participaram deste estudo 15 idosos, de ambos os sexos, com idade entre 60 e 80 anos. O programa era realizado no solo, durante 15 sessões de treinamento, com pré e pós-teste e duração de 11 semanas. Como encontrados nesse estudo, evidenciaram um aumento significativo da flexibilidade, promovendo uma redução do número de quedas entre os idosos.

Vários fatores associados à prática de EF podem influenciar no desempenho físico dos idosos, pois conforme o Colégio Americano de Medicina do Esporte (ACSM), o EF promove estímulos neuromotores, sobre a força e flexibilidade corpórea.

O Método Pilates é uma modalidade que aprimora o condicionamento físico e não apresenta restrição para pacientes com patologias osteomusculares. Ele preconiza uma harmonia dos músculos agonistas e antagonistas, ativando os músculos estabilizadores, o controle motor e o alinhamento postural, principalmente quando aplicados aos idosos (MACEDO TL, et al., 2016). 
Com o avanço da idade cronológica e as alterações corpóreas, os idosos perdem a autonomia para realizar as atividades da vida diária e afeta a sua qualidade de vida, sendo a sua capacidade funcional essencial para uma vida independente (CASTREZAN GM, et al., 2013). Nesse sentido, verificou-se com essa pesquisa, que praticantes de EF apresentavam uma capacidade funcional bem superior aos não praticantes.

No entanto, os grupos com integrantes que praticavam regularmente $E F$ (G2 e G3) não se diferenciaram nesse importante componente para a manutenção da autonomia e atividades cotidianas. Outro dado relevante verificado nessa investigação se refere a correlação entre a flexibilidade dos idosos e a melhor capacidade funcional.

Esses resultados se assemelham à diversos estudos publicados, como do Nascimento MM et al. (2019), em que avaliaram os efeitos promovidos pelo Pilates Mate na flexibilidade, marcha, mobilidade funcional, equilíbrio e força de membros inferiores - MMIl de idosas. Participaram do estudo 48 idosas, de 67 à 72 anos, subdividas em um grupo Pilates e controle.

Após 16 semanas de aplicação do treinamento, verificaram um aprimoramento da flexibilidade e equilíbrio dinâmico das idosas. Portanto, como na presente pesquisa, evidenciou-se que participantes de Pilates apresentam melhores índices de flexibilidade e capacidade funcional, que promove um melhor padrão da marcha e mobilidade de MMII.

Engers PB et al. (2015) e Costa LMRD et al. (2016) preconizam que a atividade física incorporada a rotina do idoso, principalmente aquelas voltadas para a resistência muscular e aeróbicas, influenciam na forma de minimizar as complicações do processo de envelhecimento, como perda da funcionalidade, da flexibilidade e do equilíbrio. Já no estudo desenvolvido por Vieira SCAL, et al. (2015), o método Pilates demonstrou ser um recurso efetivo para aumentar o equilíbrio estático e dinâmico. Da mesma forma, referindo-se a autonomia pessoal, Sacco ICN et al. (2005), verificaram que o Pilates mostrou ser eficaz para o aprimoramento da capacidade funcional de idosos, principalmente para realizar atividades básicas como vestir, sentar e caminhar.

Alexandrino EG et al. (2018) reforça os nossos achados relacionando a flexibilidade à capacidade funcional dos idosos. A diminuição da flexibilidade em pessoas mais velhas tende a um grande declínio da capacidade funcional e compromete as suas atividades de vida diária - AVD's. Portanto, o treinamento da flexibilidade através de EF visa desenvolver a distribuição de liquido sinovial nas articulações, melhorar a mobilidade, a força, de modo que os idosos permaneçam com níveis mais adequados.

Com relação à sintomatologia dolorosa apresentada, observou-se nos três grupos investigados, principalmente nos joelhos, segmento toracolombar, trapézio e ombros. Deve-se ressaltar que a frequência que ocorrem as manifestações álgicas se diferencia, tendo em vista que a maioria das integrantes do G1 relataram sentir dor várias vezes ao dia, enquanto grande parte das participantes do G2 e G3 relataram sentir dor de forma esporádica.

Além disso, evidenciou-se que a intensidade da dor se diferenciava de forma significativa entre os grupos, no qual as praticantes de EF apresentavam índices álgicos bem inferiores em relação às não participantes. Da mesma forma, foi observado nesta amostra, uma associação negativa entre a flexibilidade da cadeia posterior e ombros com a intensidades da dor.

Como observado nessa investigação, a presença da dor no idoso está comumente associado ao processo de envelhecimento e de patologias osteomioarticulares crônicas, que podem ser amenizadas com programas de atividades físicas que promovam a redução da obesidade, sarcopenia precoce e perda da capacidade funcional para os idosos, melhoria da qualidade de vida e autonomia (BOBBO VCD, et al., 2018; KNEIP K, et al., 2018).

Tozim BM et al. (2014), realizaram uma pesquisa para averiguar os benefícios do Pilates para a flexibilidade, qualidade de vida e dor de idosas. A amostra foi composta por 31 participantes, subdivididas em dois grupos, sendo um de Pilates $(n=14)$ e outro controle $(n=14)$. Para análise da dor foi utilizado a Escala Visual analógica e a flexibilidade pelo teste de sentar e alcançar. Como resultados parciais foi observado uma redução significativa da dor $(p=0,187)$ e manutenção da qualidade de vida. 
A prática regular do Pilates promove uma redução das manifestações álgicas em idosos, pelo aumento e manutenção da flexibilidade, com ganho de força, no qual melhora a qualidade de vida e a realização das AVDs, especialmente por atuar no corpo de forma global (ATLAN L, et al., 2009). A realização do Pilates como atividade física, estimula o sistema opioide endógeno e o não opioide (hormônio do crescimento e corticotropina), durante o treinamento e a execução dos movimentos isométricos, o que atua no organismo como um agente analgésico (TOZIM BM, et al., 2014).

\section{CONCLUSÃO}

A partir dos resultados evidenciados nesta investigação, conclui-se que idosas que praticavam regularmente exercícios físicos apresentaram melhores níveis de flexibilidade, de capacidade funcional e menores índices de manifestações álgicas do que as não praticantes. No entanto, aquelas que realizavam Pilates obtiveram melhores resultados, demonstrando assim, ser uma importante ferramenta para programas de saúde coletiva na terceira idade. Além disso, foi verificada uma associação entre a flexibilidade das idosas com maior capacidade funcional e menor indice de sintomatologia dolorosa. Esse trabalho contribuiu para uma maior discussão sobre os efeitos dos EF para idosos, tornando-se impreencedivel mais investigações nesta temática.

\section{REFERÊNCIAS}

1. ALEXANDRINO EG, et al. Flexibilidade na terceira idade: uma revisão de literatura., 2018, 23: 241.

2. ATLAN L, et al. Effect of Pilates training on people with fibromyalgia syndrome: a pilot study. Arch. Phys. Med. Rehabil., 2009; 5(90); 1983-1988.

3. ÁVILA AF, et al. Prevalência de patologias auto-relatadas por idosos pertencentes a um grupo de extensão. Anais do Salão Internacional de Ensino, Pesquisa e Extensão, 2019; 10(3).

4. BOBBO VCD, et al. Saúde, dor e atividades de vida diária entre idosos praticantes de Lian Gong e sedentários. Ciência \& Saúde Coletiva, 2018; 23(4):1151-1158.

5. CASTREZAN GM, et al. Envelhecimento e qualidade de vida: análise da produção científica da Scielo. Biblioteca Virtual de Saúde, 2013;16(1):191-199.

6. COSTA LMRD, et al. Os Efeitos do Método Pilates Aplicado à População Idosa: Uma Revisão Integrativa. Rev.Bras. Geriatr. Gerontol., Rio de Janeiro, 2016; 19(4): 695-702.

7. ENGERS PB, et al. Efeitos da prática do método Pilates em idosos: uma revisão sistemática. Rev. Bras. Reumatol., São Paulo, 2015; 56(4): 352-365.

8. FERREIRA OGI, et al. Envelhecimento Ativo e sua Relação com a Independência Funcional. Enferm., Florianopólis, 2012; 21(3): 513-518.

9. IREZ GB. The effects of different exercises on balance, fear and risk of falling among adults aged 65 and over. $J$ Anthropologist, 2014;18(1):129-34.

10. KNEIP K, et al. A influência do método Pilates solo em idosos sedentários na melhora da flexibilidade e da qualidade do sono. Revista Brasileira de Fisiologia do Exercício, 2018;17(1):38-46.

11. MACEDO TL, et al. O efeito do Método Pilates de Solo na flexibilidade de idosas. ConScientiae Saúde, 2016; 15(3): 448-456.

12. MAZO GZ, et al. Atividade física e o idoso: concepção gerontológica. Porto Alegre: Sulina, 2009; 318 p.

13. NASCIMENTO MM, et al. Efeitos de 16 semanas do método pilates mat sobre o equilíbrio, marcha, mobilidade funcional e força de idosas, 60-79 anos. Saúde, Santa Maria, 2019; 45(1):1-11.

14. REIS LA, et al. Avaliação da qualidade de vida em idosos praticantes e não praticantes do Método Pilates. C\&DRevista Eletronica da Fainor, 2011; 4(1): 38-51.

15. RIBEIRO PCC, et al. Perfil clínico e uso de serviços de saúde em idosos. Revista HUPE, 2018; 17(2): 27-36.

16. RICKLI RE, JONES CJ. Teste de aptidão física para idosos. Barueri, SP: Manole, 2008; 183 p.

17. SACCO ICN, et al. Método Pilates em revista: aspectos biomecânicos de movimentos específicos para reestruturação postural - Estudos de caso. Rev. Bras. Cien. e Mov., 2005;13: 65-78.

18. SANTOS FC, et al. Síndrome de amplificação dolorosa no idoso. Relato de caso e revisão da literatura. Rev. Dor., $2012 ; 13(2): 175-82$.

19. TOZIM M, et al. Efeito do método Pilates na flexibilidade, qualidade de vida e nível de dor em idosos ConScientiae. Saúde, 2014; 13(4): 563-570.

20. VALDANHA NETTO A, et al. Ética na Intervenção do Profissional de Educação Física com Idosos. In: VARGAS, A. (ORG.) Dimensionamento Ético da Intervenção Profissional em Educação Física. Rio de Janeiro: CONFEF, 2017.

21. VIEIRA SCAL, et al. Força Muscular Associada ao Processo de Envelhecimento. Ciências Biológicas e da Saúde, 2015; 3(1): 93-102.

22. WANDERLEY RL, et al. Impacto de multimorbidade sobre mortalidade em idosos: estudo de coorte póshospitalização. Rev. Bras. Geriatr. Gerontol., 2019; 16(3): 579-589. 